\title{
Endemischer Vitamin-D-Mangel in Mitteleuropa?
}

Im Gegensatz zu den meisten anderen Vitaminen ist der menschliche Organismus zu einer Eigensynthese von Vitamin D befähigt. Unter dem Einfluss von UV-Licht kann Vitamin $D_{3}$ (Cholecalciferol) in der Haut aus der Vorstufe 7-Dehydrocholesterin gebildet werden. Diese Eigensynthese ist unter den klimatischen Bedingungen Mitteleuropas in der Regel nicht bedarfsdeckend, was vor allem für die Wintermonate gilt. Eine kontinuierliche exogene Zufuhr von Vitamin D über die Nahrung ist daher erforderlich.

Die aktuellen Referenzwerte für die Nährstoffzufuhr (herausgegeben von den Gesellschaften für Ernährung in Deutschland, Die Eigensynthese Österreich und der Schweiz ist in der Regel D.A.CH.-Referenzwerte) nennen nicht bedarfsdeckend für das Vitamin D eine empfohlene Zufuhr von $5 \mu \mathrm{g}$ pro Tag für den erwachsenen Menschen bis 65 Jahre und von $10 \mu \mathrm{g}$ über 65 Jahre. Die höhere Zufuhr beim älteren Erwachsenen wird durch eine verminderte Fähigkeit zur Vitamin-DBildung in der Haut begründet.

Der Vitamin-D-Status kann über die Messung der Plasmakonzentration von 25-Hydroxy-Vitamin $D_{3}$ festgestellt werden. Werte zwischen 25 und $50 \mathrm{nmol} / \mathrm{l}$ werden als leichter Mangel, Werte zwischen 10 und $25 \mathrm{nmol} / \mathrm{l}$ als mittelgradiger Mangel und Werte unter $10 \mathrm{nmol} / \mathrm{l}$ als schwerer Mangel klassifiziert (Thomas: Labor und Diagnose, TH-Books GmbH, 5. Auflage 2000). Inverse Beziehungen bestehen zwischen den Serumkonzentrationen von 25-Hydroxy-Vitamin $D_{3}$ und Parathormon (PTH) und verschiedene Studien zur Ermittlung "optimaler" Serumkonzentrationen von 25-Hydroxy-Vitamin $D_{3}$ haben sich mit der Frage befasst, ab welchen Serumkonzentrationen dieses Vitamin-D-Metaboliten ein sekundärer Hyperparathyreoidismus nicht mehr auftritt. Danach sind Werte < $40 \mathrm{nmol} / \mathrm{l}$ bei jungen Mädchen mit verminderter Knochendichte der Elle und hohem PTH korreliert, Werte $<60$ nmol// gehen bei postmenopausalen Frauen mit einer erhöhten Ausscheidung von Knochenresorptionsmarkern und hohem PTH einher und erst ab Werten von ca. 100 nmol/I kann das Auftreten eines sekundären Hyperparathyreoidismus nicht mehr beobachtet werden.

Welche Werteverteilungen für 25-Hydroxy-Vitamin $D_{3}$ werden in Deutschland gefunden? Zunächst sind starke jahreszeitliche Unterschiede hervorzuheben. Untersuchungen an freiwilligen (gesunden) Probanden zeigen für den Monat Januar einen Median von 30 nmol/l, während im Juli ein Median von 65 nmol/l nachgewiesen wird. Anhand der Daten aus dem routinemäßigen Probeneingang eines deutschen Laboratoriums unterschreiten im Januar/Februar $40 \%$ der Patienten einen Grenzwert von 25 nmol// und über $80 \%$ einen Grenzwert von 50 nmol/l, während in den Monaten Juni/Juli nur $10 \%$ der Patienten den Grenzwert von $25 \mathrm{nmol} / \mathrm{I}$ unterschreiten, allerdings immer noch fast 50 \% der Patienten einen Grenzwert von 50 nmol/l. Eine besondere Häufung solcher Defizite lässt sich bei Risikogruppen, wie z.B. institutionalisierten älteren Menschen, nachweisen, die in den Wintermonaten in bis zu $90 \%$ der Fälle Werte unter 50 nmol/l aufweisen. Eine suboptimale Vitamin-D-Versorgung bzw. ein Vitamin-D-Mangel kann daher in weiten Bereichen der deutschen Bevölkerung bzw. der mitteleuropäischen Staaten nachgewiesen werden.

Die vorliegenden Zufuhrempfehlungen für Vitamin D sind offensichtlich nicht ausreichend, um in der breiten Mehrheit der Bevölkerung optimale Serumkonzentrationen von 25-Hydroxy-Vitamin $D_{3}$ einzustellen, einem sekundären Hyperparathyreoidismus vorzubeugen und um Knochenabbauprozesse zu vermindern. Hierfür dürften deutlich höhere Zufuhren erforderlich sein und eine Überarbeitung der Empfehlungen ist anzustreben.

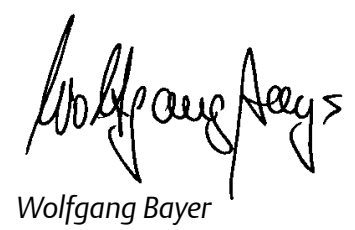

